\title{
EDITORIAL
}

\section{Canonical Wnt signaling and caveolae play a role in intervertebral disc degeneration; the continuing saga of the mysterious notochordal cell}

\author{
Mark Erwin \\ See related research by Smolders et al., http://arthritis-research.com/content/15/1/R23
}

\begin{abstract}
Over the past few decades small animal models mainly involving rodents and rabbits have been developed whereby needle puncture, stab incision or enzymatic approaches have been validated to create the degenerative disc. Although important, these models continue to be plagued by biological attributes that limit applicability to the human condition. However, the fascinating story of two naturally occurring subspecies of canine, the non-chondrodystrophic and chondrodystrophic canine, provides us with an animal model that differentially is protected from the development of degenerative disc disease. Here, Smolders and colleagues provide the first steps to understanding some of the secrets held by man's best friend.
\end{abstract}

Using microarray analysis and immunohistochemical methods, Smolders and colleagues [1] provide a fascinating and sophisticated initial investigation into the genomic regulatory differences between non-chondrodystrophic (NCD) and chondrodystrophic (CD) canine nucleus pulposus (NP). The implications of this study are notable with respect to what may well be the lynch pin concerning degeneration of the disc: the notochordal cell.

Over the past decade the use of growth factors, anticell death strategies, whole organ transplants and stem/ progenitor cells have been used in order to delay or possibly even reverse degenerative disease of the intervertebral disc (IVD) [2]. However, in addition to its

*Correspondence: mark.erwin@utoronto.ca

Divisions of Orthopaedic and Neurological Surgery, Toronto Western Hospital/ University of Toronto, 399 Bathurst Street, McLaughlin Pavilion Rm 11-408, Toronto, Ontario, Canada obvious supportive function, the IVD is an isolated, hypoxic, ischemic and immune privileged tissue compartment that poses unique challenges with respect to regenerative strategies [3]. The cells within the NP have evolved to tolerate the otherwise 'hostile' environment of low oxygen levels and tenuous nutrient and gas diffusion properties that are in turn mediated by the endplates through delicate homeostatic regulatory mechanisms $[3,4]$. However, with respect to intrinsic cellular mechanisms concerning degenerative disc disease (DDD) progression, there is still much to be learned.

With respect to understanding salient differential mechanisms concerning DDD, nature has provided an interesting anomaly whereby two naturally occurring canine subspecies are differentially protected from degenerative change; the NCD and CD canine [5,6]. These two canine sub-species are defined to a large degree by their differential susceptibility to DDD with the NCD canine NP protected from developing DDD and retaining large populations of notochordal cells (NCs) within their NP throughout life [6,7]. The CD canine on the other hand develops DDD early in life in a similar fashion to that of humans and is largely $\mathrm{NC}$-deficient $[5,6]$. A number of studies have examined NC interaction with NP cells and have determined that NCs confer a beneficial role upon the maintenance of the healthy IVD NP [7-10].

Amongst a number of important unanswered questions are the mechanisms whereby NCs persist in the NPs of NCD dogs and disappear in CD animals. Smolders and colleagues report that when $\mathrm{NC}$ populations decline during early DDD there are changes in a number of signaling pathways specifically including down-regulation of both canonical Wnt signaling and caveolin-1 expression. These changes in cellular signaling pathways suggest that the Wnt/ $\mathrm{B}$-catenin signaling cascade may be pivotal for the preservation of the NC-rich NP phenotype and perhaps also maintenance of the healthy NP phenotype. Gene expression of the important marker of 
Wnt signaling (axin2) was reported to be reduced in canine discs displaying predominantly chondrocyte-likecell (CLC) groups compared to the NC-rich and mixed groups (50:50 NCs:CLCs) in both NCD and CD dogs. However, when comparing the two sub-species irrespective of their histopathological classifications, the $C D$ dog NPs demonstrate significantly higher levels of axin2 gene expression than NCD dogs. These differences suggest that important changes in $\mathrm{Wnt} / \beta$-catenin signaling occur with the alteration of the NP from the NC-rich to the more 'chondrified' CLC disc that is more typical of the degenerative phenotype. The important and unanswered questions with respect to $\mathrm{NC}$ preservation are how these processes work and why is there an alteration in the important Wnt/ $\beta$-catenin signaling pathway? $\beta$-Catenin signaling mediates in part cadherin-related cytoskeletal organization and cell adhesion. The report by Smolders and colleagues suggests that increased Wnt/ $\beta$-catenin signaling is associated with the NP of dogs that suffer premature DDD and that the preservation of NCs seems to be integral (in an as of yet unclear fashion) to what may be homeostatic regulatory $\mathrm{Wnt} / \beta$-catenin signaling. Down-regulation of caveolin-1 gene and protein expression was observed to accompany the loss of NCs, which in turn is associated with 'chondrification' of the disc and the development of a degenerative phenotype. However, in addition to various aspects of tissue maintenance the $\mathrm{Wnt} / \beta$-catenin signaling networks are also significantly involved with the proliferation and differentiation of stem cells. Given that both of these subspecies of dog contain NP progenitor/stem cells and NP cells but varying populations of NCs, it leads one to wonder what impact the $\mathrm{Wnt} / \beta$-catenin signaling as detailed by Smolders and colleagues may have amongst this diverse cellular population and what are the implications in terms of susceptibility to DDD? Understanding the respective signaling (Wnt/ $\beta$-catenin?) pathways will be a daunting task but such knowledge might provide vital information concerning the propensity of the NP to suffer DDD.

The manuscript by Smolders and colleagues suggests that the maintenance of the NC-rich/non-degenerative NP phenotype may require appropriate homeostatic regulation involving caveolae, caveolin-1 and Wnt/Bcatenin signaling pathways. In their paper these authors indicated that the NP of wild-type mice was rich in viable $\mathrm{NCs}$, whereas the NP of caveolin-1 knockout mice contained chondroid-like matrix with mainly apoptotic, small, rounded cells. Taken together this leads one to ponder if part of the secret to avoiding DDD may lie within restoration of the homeostatic communication/ regulation between the unique cells within the IVD NP, or is DDD susceptibility a function of the peculiarities of the canine sub-species and simply a genetic anomaly [11]? It is tantalizing to consider that the human condition may actually resemble that of man's best friend-who may yet have important lessons to teach us.

\section{Abbreviations}

$C D$, chondrodystrophic; $C L C$, chondrocyte-like-cell; $D D D$, degenerative disc disease; IVD, intervertebral disc; NC, notochordal cell; NCD, nonchondrodystrophic; NP, nucleus pulposus.

\section{Competing interests}

The author declares that he has no competing interests.

Published: 22 March 2013

\section{References}

1. Smolders LA, Meij BP, Onis D, Riemers FM, Bergknut N, Wubbolts R, Grinwis GC, Houwelin M, Groot Koerkamp MJ, van Leenen D, Holstege FC, Hazewinkel HA, Creemers LB, Penning LC, Tryfonidou MA: Gene expression profiling of early intervertebral disc degeneration reveals a downregulation of canonical Wnt signaling and caveolin-1 expression: implications for development of regenerative strategies. Arthritis Res Ther 2013, 29:R23.

2. Masuda K: Biological repair of the degenerated intervertebral disc by the injection of growth factors. Eur Spine J 2008, 17(Suppl 4):S441-S451.

3. Urban JP, Smith S, Fairbank JC: Nutrition of the intervertebral disc. Spine 2004, 29:2700-2709.

4. Urban JP: The role of the physicochemical environment in determining disc cell behaviour. Biochem Soc Trans 2002, 30:858-864.

5. Hans-Jorgen $\mathrm{H}$ : A pathologic-anatomical study on disc degeneration in dog. Acta Orthopaed Scand Supp/1952, 11:1-117.

6. Braund KG: Morphological studies of the canine intervertebral disc. The assignment of the beagle to the achondroplastic classification. Res Vet Sci 1975, 9:167-172.

7. Aguiar DJ, Johnson SL, Oegema TR: Notochordal cells interact with nucleus pulposus cells: regulation of proteoglycan synthesis. Exp Cell Res 1999, 246:129-137.

8. Erwin WM, Inman RD: Notochord cells regulate intervertebral disc chondrocyte proteoglycan production and cell proliferation. Spine 2006, 31:1094-1099.

9. Erwin WM, Ashman K, O'Donnel P, Inman RD: Nucleus pulposus notochord cells secrete connective tissue growth factor and upregulate proteoglycan expression by intervertebral disc chondrocytes. Arthritis Rheum 2006, 54:3859-3867.

10. Erwin WM, Islam D, Inman RD, Fehlings MG, Tsui FW: Notochordal cells protect nucleus pulposus cells from degradation and apoptosis: implications for the pathophysiology and treatment of intervertebral disc degeneration. Arthritis Res Ther 2011, 13:R215.

11. Cappello R, Bird JL, Pfeiffer D, Bayliss MT, Dudhia J: Notochordal cell produce and assemble extracellular matrix in a distinct manner, which may be responsible for the maintenance of healthy nucleus pulposus. Spine 2006, 31:873-882.

doi:10.1186/ar4182

Cite this article as: Erwin M: Canonical Wnt signaling and caveolae play a role in intervertebral disc degeneration; the continuing saga of the mysterious notochordal cell. Arthritis Research \& Therapy 2013, 15:113. 